\title{
Quantum Complementarity of Microcavity Polaritons
}

\author{
S. Savasta, ${ }^{1}$ O. Di Stefano, ${ }^{1}$ V. Savona,${ }^{2}$ and W. Langbein ${ }^{3, *}$ \\ ${ }^{1}$ Dipartimento di Fisica della Materia e Tecnologie Fisiche Avanzate, Università di Messina, Salita Sperone 31, 98166 Messina, Italy \\ ${ }^{2}$ Institut de Physique Théorique, Ecole Polytechnique Fédérale de Lausanne (EPFL), CH-1015 Lausanne, Switzerland \\ ${ }^{3}$ Experimentelle Physik IIb, Universität Dortmund, Otto-Hahn-Str. 4, 44221 Dortmund, Germany \\ (Received 7 October 2004; revised manuscript received 16 March 2005; published 20 June 2005)
}

\begin{abstract}
We present an experiment that probes polariton quantum correlations by exploiting quantum complementarity. Specifically, we find that polaritons in two distinct idler modes interfere if and only if they share the same signal mode so that "which-way" information cannot be gathered. The experimental results prove the existence of polariton pair correlations that store the which-way information. This interpretation is confirmed by a theoretical analysis of the measured interference visibility in terms of quantum Langevin equations.
\end{abstract}

DOI: 10.1103/PhysRevLett.94.246401

PACS numbers: 71.36.+c, 42.50.Dv, 42.50.Nn, 71.35.Gg

Quantum complementarity is the essential feature distinguishing quantum from classical physics [1]. When two physical observables are complementary, the precise knowledge of one of them makes the other unpredictable. The most known manifestation of this principle is the property of quantum-mechanical entities to behave either as particles or as waves under different experimental conditions. The link between quantum correlations, quantum nonlocality, and Bohr's complementarity principle was established in a series of "which-way" experiments [25], in which the underlying idea is the same as in Young's double-slit experiment. Because of its wavelike nature, a particle can be set up to travel along a quantum superposition of two different paths, resulting in an interference pattern. If, however, a which-way detector is employed to determine the particle's path, the particlelike behavior takes over and an interference pattern is no longer observed. These experiments have brought evidence that the loss of interference is not necessarily a consequence of the back action of a measurement process [5]. Quantum complementarity is rather an inherent property of a system, enforced by quantum correlations [1]. We investigate this manifestation of quantum mechanics for cavity polaritons. Polaritons in semiconductor microcavities are hybrid quasiparticles consisting of a superposition of cavity photons and two-dimensional collective electronic excitations (excitons) in an embedded quantum well [6]. Owing to their mutual Coulomb interaction, pump polaritons generated by a resonant optical excitation can scatter resonantly into pairs of polaritons (signal and idler) [7-13]. In the low excitation limit, the polariton parametric scattering is a spontaneous process driven by vacuum-field fluctuations [9] whereas, already at moderate excitation intensity, it displays self-stimulation [12].

In this Letter we present a which-way experiment based on the polariton parametric process. Our findings demonstrate the pair correlation of the emitted signal-idler polaritons [14]. This fact, together with the possibility of ultrafast optical manipulation and ease of integration of these microdevices, holds promise for applications in quantum information science.

Polariton parametric scattering is a $\chi^{(3)}$ process in which two-pump polaritons are scattered into a signal-idlerpolariton pair, conserving total energy and momentum. The effective Hamiltonian describing the parametric polariton process assuming classical pump fields is

$$
\begin{aligned}
\hat{H}= & \sum_{\mathbf{k}} E_{\mathbf{k}} \hat{p}_{\mathbf{k}}^{\dagger} \hat{p}_{\mathbf{k}} \\
& +\sum_{\substack{\mathbf{k}, \mathbf{k}^{\prime} \\
\mathbf{k}_{s}, \mathbf{k}_{i}}}\left[G\left(\mathbf{k}, \mathbf{k}^{\prime}\right) \hat{p}_{\mathbf{k}_{s}}^{\dagger} \hat{p}_{\mathbf{k}_{i}}^{\dagger}+\text { H.c. }\right] \delta_{\mathbf{k}_{s}+\mathbf{k}_{i}, \mathbf{k}+\mathbf{k}^{\prime},}
\end{aligned}
$$

where the Bose operators $\hat{p}_{\mathbf{k}}^{\dagger}$ are the polariton creation operators, $E_{\mathbf{k}}$ is the polariton energy [Fig. 1(a)], and $G\left(\mathbf{k}, \mathbf{k}^{\prime}\right)$ contains details of the pump fields and the polariton interaction. Because of the assumption of classical pump fields, this Hamiltonian is formally equivalent to that of a $\chi^{(2)}$ parametric down-conversion [4,15]. Given a pump wave vector $\mathbf{k}_{\mathrm{p}}$, the final states of parametric processes satisfying energy and momentum conservation are represented by an "eight"-shaped curve in $\mathbf{k}$ space $[7,8]$, depicted in Fig. 1(b). A pair of final states (signal and idler) is defined by the intersections of the curve with a straight line passing through $\mathbf{k}_{\mathrm{p}}$. We denote as "idler" the modes with $k>k_{\mathrm{p}}$. The experimental scheme that we devise employs two mutually coherent pump modes of momenta $\mathbf{k}_{\mathrm{p} 1}$ and $\mathbf{k}_{\mathrm{p} 2}$ with the classical amplitudes of the twopump-polariton fields $P_{\mathbf{k}_{\mathrm{p} 1}}$ and $P_{\mathbf{k}_{\mathrm{p} 2}}$, for which $G\left(\mathbf{k}, \mathbf{k}^{\prime}\right)=$ $g P_{\mathbf{k}} P_{\mathbf{k}^{\prime}}\left(\delta_{\mathbf{k}, \mathbf{k}_{\mathrm{p} 1}}+\delta_{\mathbf{k}, \mathbf{k}_{\mathrm{p} 2}}\right)\left(\delta_{\mathbf{k}^{\prime}, \mathbf{k}_{\mathrm{p} 1}}+\delta_{\mathbf{k}^{\prime}, \mathbf{k}_{\mathrm{p} 2}}\right)$. The constant $g$ is the polariton-polariton interaction amplitude, accounting for both the Coulomb interaction and the Pauli exclusion principle [7]. Two of the four products of $\delta$ 's represent parametric processes driven by a single-pump mode. The two other terms are mixed-pump processes involving one polariton from each pump mode, whose energymomentum conservation defines the "peanut"-shaped curve illustrated in Fig. 1(b) (dashed line). Let us first 

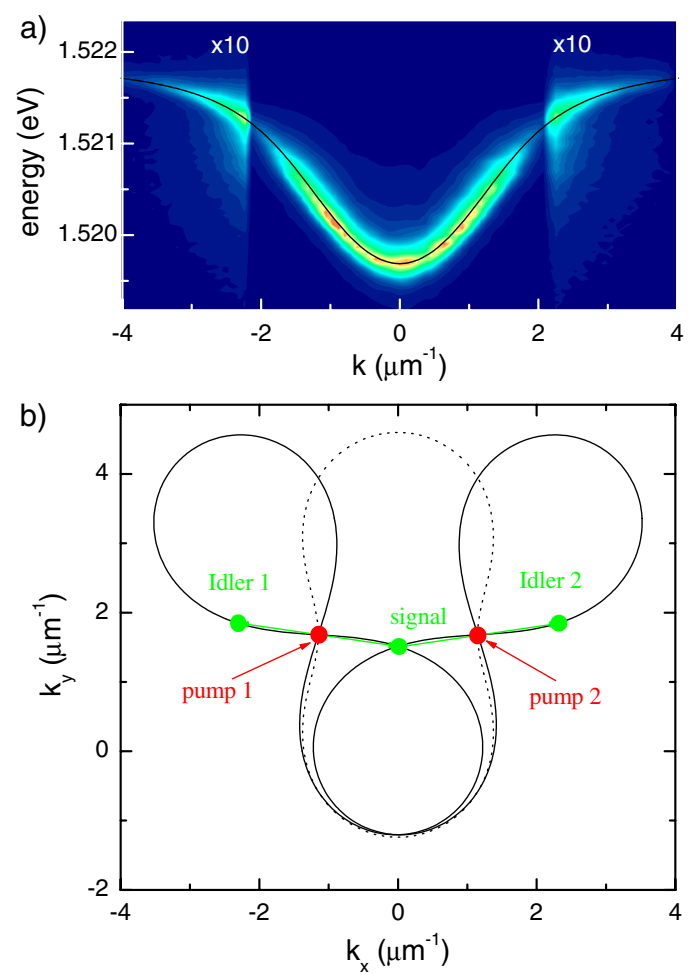

FIG. 1 (color online). (a) Lower polariton photoluminescence intensity (linear color scale) as function of energy and $|\mathbf{k}|$. Excitation is in the upper polariton branch and the detuning between the cavity resonance and the energy of the heavy-hole exciton is $-0.7 \mathrm{meV}$. The dispersion $E_{\mathbf{k}}$ (full line) calculated with a coupled-oscillator model [18] is superimposed. (b) $\mathbf{k}$-space plot of the final states fulfilling energy and momentum conservation in a two-pump parametric process. The two eight-shaped full curves represent the single-pump processes determined by the conditions $2 \mathbf{k}_{\mathrm{p} j}=\mathbf{k}_{s}+\mathbf{k}_{i}$ and $2 E_{\mathbf{k}_{\mathrm{p} j}}=$ $E_{\mathbf{k}_{s}}+E_{\mathbf{k}_{i}}$, with $j=1,2$. The dotted line describes the mixedpump process defined by $\mathbf{k}_{\mathrm{p} 1}+\mathbf{k}_{\mathrm{p} 2}=\mathbf{k}_{s}+\mathbf{k}_{i}$ and $E_{\mathbf{k}_{\mathrm{p} 1}}+$ $E_{\mathbf{k}_{\mathrm{p} 2}}=E_{\mathbf{k}_{s}}+E_{\mathbf{k}_{i}}$. Two parametric processes sharing $\mathbf{k}_{s}$, giving rise to mutual idler coherence, are indicated in green.

consider a pair produced by one pump only $\left(P_{\mathbf{k}_{\mathrm{p} 2}}=0\right)$. In the limit of low excitation intensity $\left(\tau=g\left|P_{\mathbf{k}_{\mathrm{p} 1}}\right|^{2} t \ll 1\right)$, the time evolution operator applied on the polariton vacuum state $|v\rangle$ yields the entangled polariton state $|\Psi\rangle=$ $M|v\rangle_{s, i}+\tau|1\rangle_{s}|1\rangle_{i}$, where $s$ and $i$ label a pair of signal and idler modes on the eight (i.e., $2 \mathbf{k}_{\mathrm{p} 1}=\mathbf{k}_{s}+\mathbf{k}_{i}$ ), and $M$ is a normalization constant. This quantum state shows that the parametric process produces signal-idler pair correlations, i.e., that the scattered polaritons are created in signal-idler pairs. We now consider two mutually coherent pumppolariton fields of equal amplitudes $P_{\mathbf{k}_{\mathrm{p} 2}}=P_{\mathbf{k}_{\mathrm{p} 1}} \mathrm{e}^{i \phi}$. For this scheme, pairs of parametric processes sharing the signal mode are allowed, as schematically depicted in Fig. 1(b). Such a pair of processes involves two idler modes $i 1$ and $i 2$ and one common signal mode $s$. In the limit of low excitation intensity, the time evolution operator applied on the polariton vacuum state yields in this case

$$
|\Psi\rangle=M|v\rangle_{s, i 1, i 2}+\tau|1\rangle_{s}\left(|1\rangle_{i 1}|0\rangle_{i 2}+e^{-2 i \phi}|0\rangle_{i 1}|1\rangle_{i 2}\right) .
$$

The resulting polariton population at the signal mode $\left\langle\Psi\left|\hat{p}_{\mathbf{k}_{s}}^{\dagger} \hat{p}_{\mathbf{k}_{s}}\right| \Psi\right\rangle$ is independent of $\phi$. Interference is absent due to the orthogonality of the superimposed idler states $i 1$ and $i 2$ in the parentheses. This quantum superposition stores the which-way information, each term in the sum representing one possible idler path. Interference is also absent from either idler-polariton density. A different result is found for the mutual coherence of the two idler modes, which is observable in the sum of the two idler-polariton fields. The resulting particle population is $\langle\Psi|\left(\hat{p}_{\mathbf{k}_{i 1}}^{\dagger}+\right.$ $\left.\hat{p}_{\mathbf{k}_{i 2}}^{\dagger}\right)\left(\hat{p}_{\mathbf{k}_{i 1}}+\hat{p}_{\mathbf{k}_{i 2}}\right)|\Psi\rangle=2\left\langle\Psi\left|\hat{p}_{\mathbf{k}_{i 1}}^{\dagger} \hat{p}_{\mathbf{k}_{i 1}}\right| \Psi\right\rangle[1+\cos (2 \phi)]$, showing interference because the two idler modes are pair correlated with the same signal mode, and thus even by a signal-idler coincidence measurement, no which-way information could be retrieved. We point out that similar conclusions are obtained in the regime of high excitation intensity by assuming classical signal and idler fields and random-noise terms. In this case, signal and idler phases are random but their sum phase is locked to the pump phase, which again results in a mutual idler coherence if and only if they share a common signal. As we will see below, a quantitative analysis of the measured visibility supports the quantum interpretation of the present experiment.

Polaritons at a given $\mathbf{k}$ can be observed by detecting the photons emitted at the same in-plane momentum [16], which carry the polariton amplitude and phase information. In the experiment we therefore measure the mutual interference of the two emitted idler fields indicated in Fig. 1(b) as a function of the relative pump phase $\phi$. This is accomplished by detecting two superimposed $\mathbf{k}$-resolved images of the polariton emission, one of which is preliminarily mirrored around the $k_{x}=0$ axis. The investigated sample [8] consists of a $25 \mathrm{~nm}$ GaAs $/ \mathrm{Al}_{0.3} \mathrm{Ga}_{0.7} \mathrm{As}$ single quantum well placed in the center of a $\lambda$ cavity with $\mathrm{AlAs} / \mathrm{Al}_{0.15} \mathrm{Ga}_{0.85} \mathrm{As}$ Bragg reflectors. The wide GaAs quantum well shows a negligible inhomogeneous broadening of the fundamental exciton so that polariton modes have a small broadening also at large k. The sample was held at a temperature of $5 \mathrm{~K}$. The dispersion of the lower polariton branch was determined by photoluminescence spectra as function of $|\mathbf{k}|$ [see Fig. 1(a)]. The two mutually coherent pump pulses of 1 ps Fourier-limited duration with an adjustable phase delay $\phi$ are created by splitting the exciting laser pulses (see Fig. 2) and are synchronously impinging on the microcavity with $\mathbf{k}_{\mathrm{p} 1}=\left(k_{\mathrm{p} x}, k_{\mathrm{p} y}\right)$ and $\mathbf{k}_{\mathrm{p} 2}=\left(-k_{\mathrm{p} x}, k_{\mathrm{p} y}\right)$. The pump pulses are linearly $y$ polarized, and detected is the $x$ polarized component of the emission. This configuration ensures the detection of the cocircularly polarized photons which are expected from the spin-preserving parametric process [17], while suppressing the light elastically scattered by static disorder. The pump intensity is sufficiently 


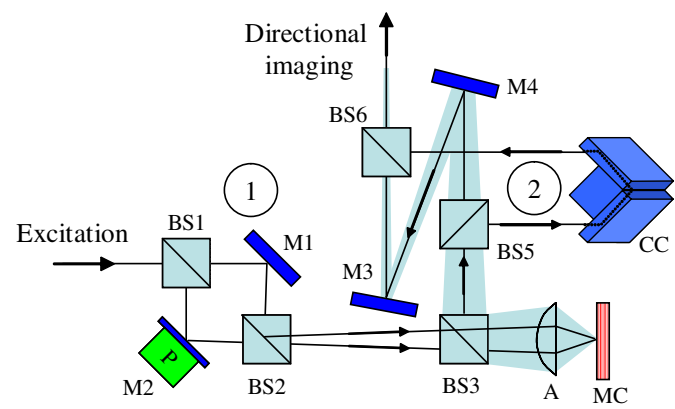

FIG. 2 (color online). Scheme of the optical setup. The two phase coherent pump pulses are created in a Mach-Zender interferometer (1) consisting of the mirrors M1, M2 and the beam splitters BS1, BS2. Their relative phase is adjustable by a piezoelectric element $(\mathrm{P})$. The pump pulses are imaged by the aspheric lens A of 0.5 numerical aperture from the mirrors onto the microcavity. The emission from the microcavity is collected by the same lens, and directed by BS3 into a second MachZender (2) which, by using a double mirror (M3, M4) in one arm and a corner cube mirror (CC) in the other one, produces interference between fields at $\mathbf{k}=\left(k_{x}, k_{y}\right)$ and $\mathbf{k}^{\prime}=\left(-k_{x}, k_{y}\right)$. All beam splitters are nonpolarizing.

low to rule out parametric processes of higher order, observed instead for higher pump intensity. The emitted photon field $\mathcal{E}_{\mathbf{k}}$ of the microcavity is directed through an interferometer creating the superposition $\mathcal{E}_{\mathbf{k}}+\mathcal{E}_{\mathbf{k}^{\prime}}$, where $\mathbf{k}=\left(k_{x}, k_{y}\right)$ and $\mathbf{k}^{\prime}=\left(-k_{x}, k_{y}\right)$.

Blocking one of the detection interferometer arms, the intensity $I_{\mathbf{k}}=\left|\mathcal{E}_{\mathbf{k}}\right|^{2}$ is measured [see Fig. 3(a)], which is proportional to the expectation value of the polariton number $N_{\mathbf{k}}=\left\langle\Psi\left|\hat{p}_{\mathbf{k}}^{\dagger} \hat{p}_{\mathbf{k}}\right| \Psi\right\rangle$. We have modeled the parametric polariton emission originating from two pumps in terms of an extension of the density matrix formalism that was previously used in the single-pump case $[7,14]$. This model shares the same physical assumptions as the simple quantum-state analysis presented here, but allows us to obtain the $\mathbf{k}$-space dependence of the polariton density. It also accounts for a finite polariton lifetime, which is mainly due to the photon escape. The calculated polariton density [Fig. 3(b)] is in qualitative agreement with the one deduced from the measured angular pattern of emission, showing that the polariton parametric process dominates in the present experimental conditions. The quantitative deviations are attributed to a deformation of the polariton dispersion occurring at moderate polariton density [7] and to details of the spin-dependent scattering not taken into account.

Using both arms of the detection interferometer, the detected intensity $\tilde{I}_{\mathbf{k}}=\left|\mathcal{E}_{\mathbf{k}}+\mathcal{E}_{\mathbf{k}^{\prime}}\right|^{2}$, proportional to the polariton number $\tilde{N}_{\mathbf{k}}=\left\langle\Psi\left|\left(\hat{p}_{\mathbf{k}}^{\dagger}+\hat{p}_{\mathbf{k}^{\prime}}^{\dagger}\right)\left(\hat{p}_{\mathbf{k}}+\hat{p}_{\mathbf{k}^{\prime}}\right)\right| \Psi\right\rangle$, depends in general on the relative excitation phase $\phi$ of the pump pulses. To understand at which $k_{x}$ the idler polaritons share the same signal mode, and thus interference is expected, we consider parametric processes driven by either one of the two-pump modes. The pump at $\mathbf{k}_{\mathrm{p} 1}$ produces the emission of polariton pairs at $\mathbf{k}$ and $2 \mathbf{k}_{\mathrm{p} 1}-\mathbf{k}$, respec-
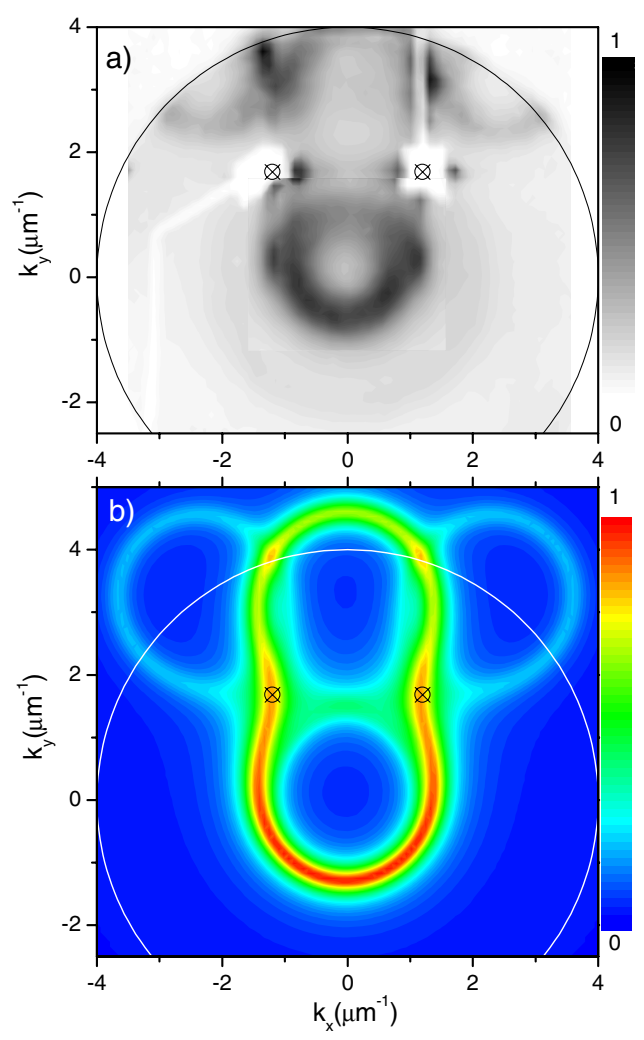

FIG. 3 (color online). Polariton density vs k. The circles indicate the experimentally accessible $\mathbf{k}$ range, and the crosses the positions of the pump pulses. (a) Measured for pulsed excitation of $\approx 9 \mathrm{~nJ} / \mathrm{cm}^{2}$, corresponding to an excited polariton density of $\approx 3 \times 10^{9} \mathrm{~cm}^{-2}$. The white shadows are masks blocking the pump beams. (b) Simulated for cw excitation using the experimentally determined polariton dispersion and pump momenta, and a constant polariton energy linewidth $\gamma=0.1 \mathrm{meV}$.

tively. Equivalently, the pump at $\mathbf{k}_{\mathrm{p} 2}$ produces pairs of $\mathbf{k}^{\prime}$ and $2 \mathbf{k}_{\mathrm{p} 2}-\mathbf{k}^{\prime}$. By construction, the modes at $2 \mathbf{k}_{\mathrm{p} 1}-\mathbf{k}$ and $2 \mathbf{k}_{\mathrm{p} 2}-\mathbf{k}^{\prime}$ have the same $y$ component but opposite $x$ components $\pm\left(2 k_{\mathrm{p} x}-k_{x}\right)$. Consequently, idler modes at $\mathbf{k}$ and $\mathbf{k}^{\prime}$ share the same signal mode if and only if $k_{x}=2 k_{\mathrm{p} x}$. We determine the visibility from the measurements using $V_{\mathbf{k}}=\sqrt{2\left[\left\langle\left(\tilde{I}_{\mathbf{k}}\right)^{2}\right\rangle_{\phi} /\left\langle\tilde{I}_{\mathbf{k}}\right\rangle_{\phi}^{2}-1\right]}$, where $\langle\cdots\rangle_{\phi}$ denotes the average over $\phi$. The visibility for the parametric process depicted in Fig. 1(b) is given in Fig. 4(a), showing that interference is actually observed at and only at the expected region in $\mathbf{k}$ space. The measured interference between the two idler polaritons shows a $\cos (2 \phi)$ dependence [see Fig. 4(b)], as predicted by Eq. (2). Additionally, in agreement with the above analysis, the signal at $k_{x}=0$ (dotted line) displays no interference, even though it is created by a superposition of contributions from both pumps.

As our experiment uses pulsed excitation, the idler interference has a distinct dynamics during the buildup and the decay of the parametric scattering [8]. In Fig. 4(c) we show the measured interference visibility as a function of time, together with the idler intensity. The 

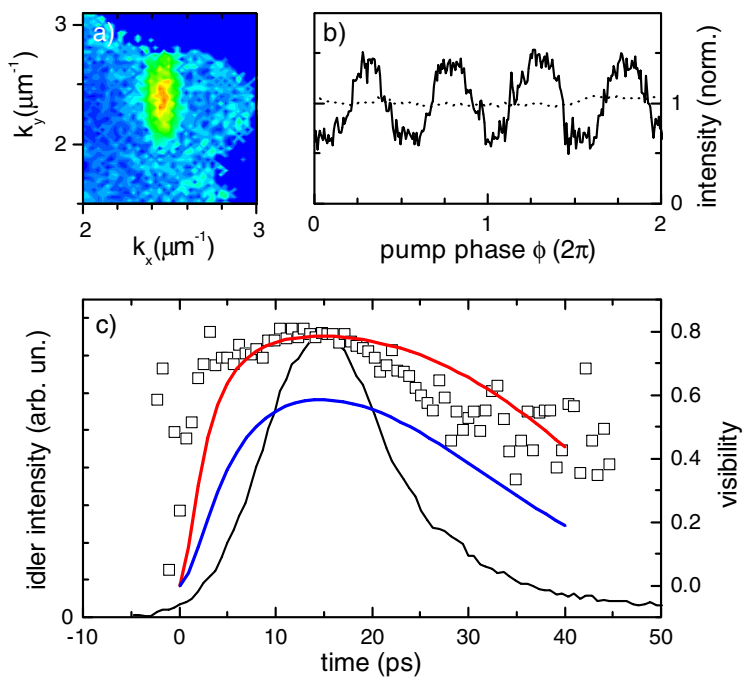

FIG. 4 (color online). Measured interference between parametric emission at $\mathbf{k}$ and $\mathbf{k}^{\prime}$. (a) $\mathbf{k}$ resolved idler interference visibility (color scale 0 to 0.5 ). (b) Measured $\tilde{I}_{\mathbf{k}}$ at $\mathbf{k}=$ $(2.4,2.4) \mu \mathrm{m}^{-1}$ (full line) and at $\mathbf{k}=(0.0,1.0) \mu \mathrm{m}^{-1}$ (dotted line) vs pump phase $\phi$. (c) Measured time-resolved idler intensity (full line, left scale) and interference visibility (squares, right scale). In color the classical (blue) and quantum (red) theoretical predictions of the visibility.

visibility reaches 0.8 soon after the pump pulse, much earlier than the maximum in the idler-polariton number. A coincidence detection of signal-idler pairs could directly assess the quantum origin of the interference [3], but was not available for the present work. We therefore need to consider that interference at high field intensity could be equally accounted for by a classical parametric model including random-noise driving terms [15]. The present measurement was performed at the onset of the selfstimulated regime, corresponding to an excitation intensity of $40 I_{0}$ according to Ref. [8]. In order to assess the nature of the measured interference, we have to compare it with the quantum and the classical prediction. This is done using the quantum Langevin model [15], generalized to include two idler modes and a time-dependent pump. We denote by $N_{0}(t)$ the time-dependent signal-polariton population, and by $N_{1,2}(t)=\langle\Psi|\left(\hat{p}_{\mathbf{k}_{i 1}}^{\dagger} \pm e^{-2 i \phi} \hat{p}_{\mathbf{k}_{i 2}}^{\dagger}\right)\left(\hat{p}_{\mathbf{k}_{i 1}} \pm\right.$ $\left.e^{-2 i \phi} \hat{p}_{\mathbf{k}_{i 2}}\right)|\Psi\rangle / 2$ the idler-polariton superpositions of which only $N_{1}$ is influenced by parametric coupling. The analytical solution reads

$$
\begin{aligned}
N_{0,1}(t)= & e^{-2 \gamma t}\left\{N_{0,1}(0) \cosh ^{2}[F(t, 0)]+\left[N_{1,0}(0)+1\right]\right. \\
& \left.\times \sinh ^{2}[F(t, 0)]\right\}+2 \gamma \int_{0}^{t} e^{-2 \gamma\left(t-t^{\prime}\right)} \\
& \times\left\{\sinh ^{2}\left[F\left(t, t^{\prime}\right)\right]\left(n_{1,0}+1\right)\right. \\
& \left.+\cosh ^{2}\left[F\left(t, t^{\prime}\right)\right] n_{0,1}\right\} d t^{\prime}, \\
& N_{2}(t)=e^{-2 \gamma t} N_{2}(0)+\left(1-e^{-2 \gamma t}\right) n_{2} .
\end{aligned}
$$

Here $\gamma$ is the polariton lifetime, $n_{j}(j=0,1,2)$ are the populations of the thermal reservoir acting as a noise source term, and $F\left(t, t^{\prime}\right)=\int_{t}^{t^{\prime}} \sqrt{2} g P_{\mathbf{k}_{p 1}}^{2}\left(t^{\prime \prime}\right) d t^{\prime \prime}$. We adjust the pump amplitude to reproduce the measured variation of the idler intensity as a function of the pump intensity $\left(1 / I_{\mathbf{k}}\right) d I_{\mathbf{k}} / d I_{P}$, where $I_{P}=\left|P_{\mathbf{k}_{p 1}}\right|^{2}$. Both the noise terms $n_{0,1}$ and the initial conditions $N_{0,1}(0)$ are taken equal to 0.2 , which reproduces the measured photoluminescence intensity in a momentum region such that $|\mathbf{k}|=\left|\mathbf{k}_{i 1}\right|$ but parametric scattering is absent. With these values, the estimated peak idler-polariton occupation number per mode is $N_{\mathbf{k}_{i 1}}=N_{\mathbf{k}_{i 2}} \sim 2$. The calculated visibility is plotted as a red curve in Fig. 4(c). From the same model, by neglecting the Bose commutator terms $(N+1 \rightarrow N)$, we obtain the classical prediction (blue curve). The comparison of the two results with the measurement suggests the quantum origin of the measured interference.

In conclusion, we have devised and performed an experiment where pair-correlated states of electronic excitations (polaritons) in a semiconductor system were produced. A quantitative analysis of the measured visibility supports the quantum interpretation of the present experiment. This result opens the possibility of producing many-particle entangled states of light-matter waves in a semiconductor, extending further the perspective of using solid-state microdevices for the implementation of quantum information technology.

We are grateful to A. Callegari, B. Deveaud, R. Girlanda, A. Quattropani, P. Schwendimann, and R. Zimmermann for discussions and suggestions. W.L. acknowledges support by U. Woggon. The sample was grown by J. Riis Jensen at the Research Center COM and the Niels Bohr Institute, Copenhagen University. V.S. acknowledges funding from the Swiss National Science Foundation through Project No. 620-066060.

*Present address: Department of Physics and Astronomy, Cardiff University, 5 The Parade, Cardiff CF24 3YB, United Kingdom.

[1] M. O. Scully et al., Nature (London) 351, 111 (1991).

[2] T. J. Herzog et al., Phys. Rev. Lett. 75, 3034 (1995).

[3] X. Y. Zou et al., Phys. Rev. Lett. 67, 318 (1991).

[4] L. Mandel, Rev. Mod. Phys. 71, S274 (1999).

[5] Y.-H. Kim et al., Phys. Rev. Lett. 84, 1 (2000).

[6] C. Weisbuch et al., Phys. Rev. Lett. 69, 3314 (1992).

[7] C. Ciuti et al., Phys. Rev. B 63, 041303R (2001).

[8] W. Langbein, Phys. Rev. B 70, 205301 (2004).

[9] S. Savasta et al., Phys. Rev. Lett. 77, 4736 (1996).

[10] S. Savasta et al., Phys. Rev. Lett. 90, 096403 (2003).

[11] R. Houdré et al., Phys. Rev. Lett. 85, 2793 (2000).

[12] R. M. Stevenson et al., Phys. Rev. Lett. 85, 3680 (2000).

[13] J. Erland et al., Phys. Rev. Lett. 86, 5791 (2001).

[14] P. Schwendimann et al., Phys. Rev. B 68, 165324 (2003).

[15] M.O. Scully and M.S. Zubairy, Quantum Optics (Cambridge University Press, Cambridge, England, 1997).

[16] V. Savona et al., Phys. Rev. B 53, 13051 (1996).

[17] P. G. Savvidis et al., Phys. Rev. Lett. 84, 1547 (2000).

[18] W. Langbein, J. Phys. Condens. Matter 16, S3645 (2004). 